\title{
Mechanisms of ischaemic stroke after chickenpox
}

\author{
V Ganesan, F J Kirkham
}

\begin{abstract}
Ischaemic stroke is a recognised complication of chickenpox. Seven cases of ischaemic stroke in children after recent varicella infection are discussed in detail to emphasise that there are several mechanisms by which this may arise. (Arch Dis Child 1997;76:522-525)
\end{abstract}

Keywords: chickenpox; stroke; protein S

Chickenpox is a common childhood illness, predominantly affecting children under the age of 10 years. Central nervous system complications are well recognised and include several reports of ischaemic stroke affecting both adults and children. ${ }^{1-4}$

As part of a questionnaire given to parents of children with ischaemic stroke (the main aim of which was to explore functional outcome), we undertook a survey to investigate how frequently ischaemic stroke in children occurred within six months of chickenpox. The results of this survey are discussed and seven cases are described in detail.

\section{Case reports}

The patient details are summarised in table 1 .

CASE 1

A girl aged 18 months developed an acute left hemiparesis two months after otherwise uncomplicated chickenpox. Magnetic resonance imaging (MRI) of the brain showed infarction

Neurosciences Unit, Institute of Child Health (UCL), London V Ganesan

F J Kirkham

Correspondence to: Dr V Ganesan, Newcomen Centre, Guy's Hospital, St Thomas Street, London SE1 9RT.

Accepted 10 February 1997 in the territory of the right middle cerebral artery (MCA) and magnetic resonance angiography (MRA) showed loss of signal in the proximal right MCA, suggestive of stenosis. She had no prothrombotic abnormalities. The MCA lesion was unchanged on MRA 18 months after the stroke. She is on long term aspirin treatment and has made a good functional recovery, with no recurrent events.

Table 1 Patient details

\begin{tabular}{llll}
\hline $\begin{array}{l}\text { Patient } \\
\text { No }\end{array}$ & $\begin{array}{l}\text { Interval between } \\
\text { chickenpox and } \\
\text { stroke }\end{array}$ & Cerebrovascular abnormality & Prothrombotic abnormality \\
\hline 1 & 2 Months & $\begin{array}{l}\text { MCA stenosis; persistent at 18 } \\
\text { months }\end{array}$ & None \\
2 & 6 Weeks & MCA stenosis & None \\
3 & 4 Months & $\begin{array}{l}\text { MCA occlusion acutely; MCA } \\
\text { stenosis 6 months later }\end{array}$ & None \\
4 & 1 Week & Moyamoya & None \\
5 & 2 Months & MCA stenosis; persistent at 1 & Low total protein S \\
6 & year & $\begin{array}{l}\text { Low free protein S } \\
\text { Now free and total protein S, } \\
\text { protein C, and } \\
\text { antithrombin III }\end{array}$ \\
& 1 Week & PCA occlusion &
\end{tabular}

$\mathrm{MCA}=$ middle cerebral artery; $\mathrm{PCA}=$ posterior cerebral artery.
CASE 2

A 5 year old boy presented with a right hemiparesis six weeks after chickenpox. MRI showed infarction in the left MCA territory, with evidence of left MCA stenosis on MRA. A prothrombotic screen (levels of protein C,S antithrombin III, heparin cofactor II, plasmingoen, activated protein $\mathrm{C}$ resistance ratio, and investigations to detect the factor $\mathrm{V}$ Leiden mutation or an antiphospholipid antibody) at the time of the ictus was normal. He has been treated with low dose aspirin; however, although he has only been followed up for three months, he has had a transient ischaemic event during this time, without any evidence of progression of his vascular lesion, and has significant dystonia affecting his hemiparetic hand.

CASE 3

A 6 year old girl developed a left hemiparesis four months after having chickenpox. MRI of the brain showed an infarct in the territory of the right MCA; the right MCA was occluded on contrast cerebral angiography. A full prothrombotic screen (as in case 2) was normal. She has a dense residual hemiparesis but has had no recurrent events. She is on long term low dose aspirin treatment. Repeat MRA six months after the ictus showed MCA stenosis with some distal flow.

CASE 4

A 3.5 year old Iranian boy developed an acute left hemiparesis one week after the onset of chickenpox. He was seen by us six months later. MRI of the brain revealed a mature infarct in the right parietal lobe with multiple small foci of infarction in the right frontal white matter. MRA demonstrated reduced flow in both carotid siphons with prominent collateral vessels. A contrast cerebral angiogram confirmed the diagnosis of bilateral moyamoya (large vessel occlusion with collateral) affecting both anterior and posterior circulations. All investigations for additional risk factors for stroke, including a full prothrombotic screen six months after the acute presentation, were normal. He has had several transient ischaemic events in the 16 months after the initial stroke and has some persistent difficulties with coordination and language. He is being treated with long term low dose aspirin treatment.

CASE 5

A 5 year old boy developed an acute right hemiparesis two months after otherwise uncomplicated chickenpox. The neurological deficit resolved within 16 hours; however he had evidence of left striatocapsular infarction on MRI. His total protein S concentration was 
low at the time of the stroke but normalised within three months. He had no other risk factors for stroke. MRA a year later showed persistent MCA stenosis. He has no residual neurological deficits and is being treated with warfarin.

CASE 6

A 6 year old boy complained of sudden visual impairment a week after developing chickenpox. He then developed tonic-clonic movements involving the right hand and foot, lasting two minutes. There was no loss of consciousness. He was drowsy for an hour after this episode and went on to have three further events within the next six hours. In hospital he was noted to have a left homonymous hemianopia and a healing vesicular rash. Computed tomography with contrast showed a right occipital infarct and an electroencephalogram showed widespread slow activity over the right hemisphere. No cerebrovascular imaging was performed.

Routine haematology and biochemistry were unremarkable. The cerebrospinal fluid was acellular with normal chemical composition. Antivaricella zoster virus IgM was detected in his blood. Cardiac evaluation was unremarkable. Although his basic coagulation profile (prothrombin time, activated partial thromboplastin time, thrombin time, and fibrinogen) was normal, measurement of the anticoagulant proteins on the day of admission showed a low concentration of free protein S at 57\% (normal range $75-125 \%$ ). The total protein $S$ concentration was normal at $94 \%$ (normal range $75-125 \%$ ) as were those of protein C, antithrombin III, heparin cofactor II, and plasminogen; platelet aggregation studies were also normal. His complement profile at the time was normal, in particular the level of C4 was not raised. The free protein $S$ normalised after 10 days. He made a good functional recovery and was discharged on low dose aspirin. He has had no recurrent events.

CASE 7

A 8 month old girl became acutely encephalopathic one month after developing chickenpox. She rapidly deteriorated after presentation to hospital, becoming shocked with marked cardiorespiratory compromise. Venoarterial extracorporeal membrane oxygenation (ECMO) was started. Chest radiography showed a left pneumothorax and right empyema, from which Staphylococcus aureus was isolated. Shortly after being stabilised on ECMO she developed a florid purpuric rash affecting the whole body but particularly marked in the extremities. Haematological investigation showed evidence of a consumptive coagulopathy with thrombocytopenia and extremely low concentrations of protein C, protein S, and antithrombin III. She was treated with repeated infusions of fresh frozen plasma, protein C, and antithrombin III concentrates and a single dose of intravenous immunoglobulin. The cutaneous lesions began to improve, but she went on to have amputation of the tips of the digits on the left hand due to necrosis. The levels of the anticoagulant proteins only normalised after 14 days.

On the sixth day she had persistent bradycardia and hypertension. Paralysing drugs were withdrawn and neurological examination revealed a left hemiparesis. MRI of the brain showed evidence of infarction in the territory of the right posterior cerebral artery (PCA). The was no signal from the right PCA on MRA, suggesting it was occluded. Echocardiography and electrocardiography were normal. ECMO was discontinued on day 7 and the vessels which had been cannulated for vascular access were reconstructed. She recovered well and despite a dense left hemiparesis was developmentally age appropriate at the time of discharge on day 21 .

\section{Results of questionnaire survey}

In a retrospective questionnaire survey of parents of 58 children with stroke, 10 children (17\%) were reported to have had chickenpox within the six month period preceding the stroke, including three of the cases discussed in detail above. Five of these children had a prothrombotic screen at the time of the event and none had any abnormalities. In addition to three patients discussed in detail above, one child had evidence of occlusion of the anterior inferior cerebellar artery on contrast cerebral angiography. Of the remaining six, one had structural cardiac disease, one had meningitis, and final diagnoses were hemiplegic migraine in one case and acute disseminated encephalomyelitis in another. The remaining two children, one of whom did not have any cerebrovascular imaging, had cerebral infarction on MRI with no risk factors identified.

\section{Discussion}

Although cerebral infarction after herpes zoster ophthalmicus is well recognised, ${ }^{56}$ the literature on ischaemic stroke after chickenpox in childhood is confined to a number of case reports ${ }^{1-3}$ and this complication has been said to be rare. However, a Japanese study in 1990 suggested that stroke complicates 1:6500 cases of childhood varicella infection. ${ }^{7}$ The data from our questionnaire survey suggest that the association is common and should be actively sought in order to direct investigation towards identification of possible vascular or thrombotic abnormalities. It cannot, however, be inferred that the preceding chickenpox was significant in all cases and in the seven cases identified from this survey, but not discussed in detail, there appeared to be no clear link between the two events. The literature suggests that stroke is a delayed complication of chickenpox; although this was true in five of our seven cases, in the other two patients (cases 4 and 6) cerebral infarction occurred within the first week of the illness.

Previous reports have focused on cerebrovascular disease as the primary mechanism of stroke in these cases. Where cerebral angiography is performed, there is typically occlusion or stenosis of proximal large vessels, ${ }^{1}$ well illustrated by our patients. Vasculitic changes, irregular stenosis, and beading involving multi- 
ple vessels have also been described, ${ }^{23}$ reminiscent of granulomatous angiitis. It has been suggested that such lesions represent the effects of a vasculitic reaction to viral invasion along the ophthalmic division of the trigeminal nerve. $^{2-6}$ The arteries innervated by this nerve correspond to the typical distribution of vascular involvement in this condition. Pathological studies in cases of herpes zoster ophthalmicus have demonstrated virus particles in the media but not the endothelium of vessels, lending further support to this hypothesis. ${ }^{8}$ However, it is not always possible to demonstrate either viral particles or viral antigens in affected vessels $^{6}$ and patients without facial or corneal lesions, or indeed any cutaneous lesions, ${ }^{4}$ may have typical vascular disease. Alternative mechanisms that have been put forward include spread via the bloodstream or cerebrospinal fluid or an immune mediated vascular reaction secondary to distant infection. ${ }^{8}$

Moyamoya, as observed in case 4 , has not previously been described after varicella infection. Moyamoya is an angiographic description of large vessel occlusion with collateral formation and thus can be the result of various pathological processes. Patients are prone to recurrent ischaemic events. Children who initially have isolated large vessel stenosis may progress to develop moyamoya but the factors which determine progression or resolution of vascular disease in individual cases remain unclear. Bodensteiner et al suggested that the cerebrovascular lesions in chickenpox could be mediated by sympathetic stimulation due to the local irritant effect of the varicella lesions in the region of the superior cervical ganglion. ${ }^{1}$ Such a mechanism has also been put forward for the aetiology of idiopathic moyamoya. ${ }^{9}$

It has been stated that the risk of recurrent events in stroke after varicella is low and that vascular lesions seen after varicella improve over time. ${ }^{12}$ However, two of our patients (cases 2 and 4 ) had recurrent transient ischaemic attacks within 18 months of the original stroke. In the four children with cerebrovascular lesions whom we have followed up over at least six months, all have had persistent vascular abnormalities and some improvement was seen in only one patient (case 3). Long term follow up should therefore include assessment for evidence of persistent or progressive vascular disease; this may be done non-invasively with serial MRA studies.

Although systemic thrombotic complications, such as purpura fulminans, complicating chickenpox are well recognised, the role of thrombosis in stroke after chickenpox has previously received little attention. Eidelberg et al demonstrated histological evidence of thrombotic vascular occlusion, without evidence of a vasculitic reaction, in three patients with herpes zoster opthalmicus and contralateral hemiparesis, involving proximal large branches of the circle of Willis. ${ }^{5} \mathrm{He}$ suggested that virus mediated endothelial injury promotes local thrombosis and vascular occlusion, as viral antigen was demonstrated in the vessel wall in these patients.
Levin et al recently demonstrated that in children with purpura fulminans complicating chickenpox, deficiencies of both free and total protein $S$ were due to circulating autoantibodies directed against protein $S$, with immune complex formation and increased clearance of protein $\mathrm{S}$ from the circulation. ${ }^{10}$ Their series included a child with an acute encephalopathy, associated with a low density lesion on computed tomography, similar to case 6 in our group. There is evidence to suggest that transient deficiencies of both protein $S$ and protein $\mathrm{C}$ may complicate varicella with a less florid clinical picture. Nguyen et al described a patient with uncomplicated varicella who had low concentrations of free and total protein $S$ but no thrombotic manifestations and another, similar to case 5, with MCA territory infarction, low concentrations of free protein $S$, and no other manifestations of thrombosis. ${ }^{11} \mathrm{Al}-$ though it could be argued that free protein $S$ deficiency in this context is the result of increased concentrations of $\mathrm{C} 4 \mathrm{~b}$ (the protein to which protein $S$ is normally bound), this may still be of functional significance as it is the free fraction of protein $S$ which exerts its anticoagulant effect. One patient (case 6) was shown to have normal concentrations of C4 suggesting that in his case the free protein $\mathrm{S}$ deficiency was due to increased consumption rather than increased binding to $\mathrm{C} 4 \mathrm{~b}$; it is unclear whether this was the cause or effect of severe thrombosis.

The optimal treatment of cerebrovascular lesions associated with varicella remains unclear. Reports include the use of steroids and antiplatelet agents and improvement of vasculitic changes has been documented. ${ }^{12}$ It is uncertain whether such an improvement was the effect of treatment as up to $50 \%$ of cerebrovascular lesions in childhood stroke will improve or resolve within a year without specific treatment. ${ }^{12}$ Although the use of steroids and immunosuppressants may be of benefit in cases of isolated cerebral angiitis, ${ }^{13}$ there is no evidence to support the use of such therapy in cases of focal cerebrovascular disease. The risk of inducing resurgent herpes zoster infection would also make such therapy unattractive in these cases. The role of antiviral agents in this situation remains unresolved ${ }^{6}$ but the variable temporal relationship between infection and onset of neurological symptoms suggests that such treatment may have a limited role. Given the persistence of vascular abnormalities in some cases, we have elected to offer these children anticoagulant or antiplatelet therapy in an effort to reduce the risks of recurrence.

Acute treatment with heparin and fresh frozen plasma should be considered for patients with prothrombotic abnormalities. Such treatment appears to be effective in cases where there is peripheral thrombosis; it is more difficult to assess its efficacy in cases of cerebral arterial thrombosis where permanent damage may have occurred before clinical presentation.

The association between ischaemic stroke in childhood and infection with varicella zoster virus appears to be significant; both cerebrov- 
ascular disease and thrombotic mechanisms appear to be implicated. Although prothrombotic abnormalities are usually transient, vascular abnormalities may be persistent. This may account for the fact that some patients go on to have recurrent events. A history of recent chickenpox should be sought in children presenting with stroke and investigation should be directed towards identification of prothrombotic and vascular abnormalities as these have implications for both acute and long term management. Recognition of stroke and residual disability as significant complications of chickenpox may add weight to the arguments for immunising children against varicella zoster.

VG and FJK were supported by the Wellcome Trust.

1 Bodensteiner JB, Hille MR, Riggs JE. Clinical features of vascular thrombosis following varicella. Am $\mathcal{F}$ Dis Child 1992;146:100-3.

2 Shuper A, Vining EPG, Freeman JM. Central nervous system vasculitis after chickenpox-cause or coincidence? Arch Dis Child 1990;65:1245-8.
3 Kamholz J, Tremblay G. Chickenpox with delayed contralateral hemiparesis caused by cerebral angiitis. Ann Neurol ateral hemiparesis

4 Vichez-Padilla JJ, Redon J, Ruiz A, Lopez-Aldeguer. CNS varicella-zoster vasculitis. Arch Neurol 1982;39:785.

5 Eidelberg D, Sotrel A, Horoupian DS, Neumann PE, Pumarola-Sune T, Price RW. Thrombotic cerebral vasculopathy associated with herpes zoster. Ann Neurol 1986;19: 7-14.

6 Hilt DC, Buchholz D, Krumholz A, Weiss H, Wolinsky JS. Herpes zoster ophthalmicus and delayed contralateral hemiparesis caused by cerebral angiitis: diagnosis and management approaches. Ann Neurol 1983;14:543-53.

7 Ichiyama T, Houdou S, Kisa T, Ohno K, Takeshita K. Varicella with delayed hemiplegia. Pediatr Neurol 1990;6:27981.

8 Linnemann CC, Alvira MM. Pathogenesis of varicellazoster angiitis in the CNS. Arch Neurol 1980;37:239-40.

9 Suzuki J, Kodama N. Moyamoya disease-a review. Stroke 1983;14:104-9.

10 Levin M, Elsey BS, Louis J, Cohen H, Young L, Heyderman RS. Postinfectious purpura fulminans caused by an antibody directed against protein S. F Pediatr 1995;127: 355-63.

11 Nguyen P, Reynaud J, Pouzol P, Munzer M, Richard O, Francois P. Varicella and thrombotic complications associated with transient protein $\mathrm{C}$ and protein $\mathrm{S}$ deficiencies in children. Eur F Pediatr 1994;154:646-9.

12 Shirane R, Sato S, Yoshimato T. Angiographic findings of ischaemic stroke in children. Childs Nerv Syst 1992;8:4326.

13 Moore PM. Diagnosis and management of isolated angiitis of the central nervous system. Neurology 1989;39:167-73. 